Article

\title{
Existence and Stability Results on Hadamard Type Fractional Time-Delay Semilinear Differential Equations
}

\author{
Nazim Mahmudov ${ }^{1, *(1)}$ and Areen Al-Khateeb ${ }^{2}$ \\ 1 Department of Mathematics, Eastern Mediterranean University, Famagusta 99628, T.R. North Cyprus, \\ Mersin 10, Turkey \\ 2 Department of Support Courses, Jadara University, P.O.Box 733, Irbid 21110, Jordan; areen.k@jadara.edu.jo \\ * Correspondence: nazım.mahmudov@emu.edu.tr
}

Received: 6 July 2020; Accepted: 19 July 2020; Published: 30 July 2020

check for updates

\begin{abstract}
A delayed perturbation of the Mittag-Leffler type matrix function with logarithm is proposed. This combines the classic Mittag-Leffler type matrix function with a logarithm and delayed Mittag-Leffler type matrix function. With the help of this introduced delayed perturbation of the Mittag-Leffler type matrix function with a logarithm, we provide an explicit form for solutions to non-homogeneous Hadamard-type fractional time-delay linear differential equations. We also examine the existence, uniqueness, and Ulam-Hyers stability of Hadamard-type fractional time-delay nonlinear equations.
\end{abstract}

Keywords: mittag-leffler type matrix function; Hadamard type derivative; Ulam-Hyers stability

Mathematical descriptions of the models described through differential equations with derivatives of non-integer orders have proved to be a very useful instrument for modeling in viscoelasticity, stability theory, controllability theory, and other related fields. Time-delays are often related with physico-chemical processes, electric networks, hydraulic networks, heredity in population growth, the economy and other related industries. In general, a peculiarity of these mathematical models is that the rate of change of these processes depends on past history. Differential systems describing these models are called time-delay differential equations. The qualitative theory of linear time-delay equations is well investigated. Recently, time-delay differential equations have been considered. In [1-9] authors derived the exact expressions for solutions of linear continuous and discrete delay equations by proposing the concept of delayed matrix functions. On the other hand, stability concepts and relative controllability problems of linear time-delay differential equations were investigated in [10-17].

The unification of differential equations with delay and differential equations with fractional derivatives is provided by differential equations including both delay and non-integer derivatives, so called time-delay fractional differential equations. In applications, this unification is useful for creating useful models of some systems with memory. One can notice that works in this field involve Riemann-Liouville and Caputo type fractional derivatives. For the literature on the related field of fractional time-delay equations of Caputo type and Riemann-Liouville type, we refer the researcher to [13-23].

Besides these derivatives, there is another fractional derivative, involving the logarithmic function: the so-called Hadamard type fractional derivative. Details of the Hadamard type fractional integral and derivative can be found in [24]. Recent results on the existence and uniqueness of solution for fractional differential equations in Hadamard sense can be found in [25-32]. 
In ([24], p. 235) it is shown that a solution of a Hadamard type fractional linear system

$$
\begin{aligned}
\left({ }^{H} D_{1^{+}}^{\alpha} y\right)(t) & =\lambda y(t)+f(t), t \in(1, T], h>0, \\
\left({ }^{H} I_{1^{+}}^{1-\alpha} y\right)\left(1^{+}\right) & =a \in \mathbb{R}, \lambda \in \mathbb{R}, 0<\alpha<1,
\end{aligned}
$$

has the form

$$
y(t)=a(\ln t)^{\alpha-1} E_{\alpha, \alpha}\left[\lambda(\ln t)^{\alpha}\right]+\int_{1}^{t}\left(\ln \frac{t}{s}\right)^{\alpha-1} E_{\alpha, \alpha}\left[\lambda\left(\ln \frac{t}{s}\right)^{\alpha}\right] f(s) \frac{d s}{s} .
$$

However, we find that there exists only one [33] work on the representation of explicit solutions of Hadamard type fractional order delay linear differential equations. In [33] authors studied the Hadamard type fractional linear time-delay system

$$
\begin{aligned}
\left({ }^{H} D_{1^{+}}^{\alpha} y\right)(t) & =\mathbb{B} y(t-h), t \in(1, T], h>0, \\
y(t) & =\varphi(t), \quad 1 \leq t \leq h, \\
\left({ }^{H} I_{1^{+}}^{1-\alpha} y\right)\left(1^{+}\right) & =a \in \mathbb{R}^{n},
\end{aligned}
$$

where $\mathbb{B}$ is a constant $n \times n$ square matrix.

Motivated by the above researches, we investigate a new class of Hadamard-type fractional delay differential equations. We consider an explicit representation of solutions of a Hadamard type fractional time-delay differential equation of the following form by introducing a new delayed M-L type function with logarithm

$$
\left\{\begin{array}{c}
\left({ }^{H} D_{1^{+}}^{\alpha} y\right)(t)=\mathbb{A} y(t)+\mathbb{B} y\left(\frac{t}{h}\right)+f(t), t \in(1, T], h>0 \\
y(t)=\varphi(t), \quad \frac{1}{h}<t \leq 1 \\
\left({ }^{H} I_{1^{+}}^{1-\alpha} y\right)\left(1^{+}\right)=a \in \mathbb{R}^{n}
\end{array}\right.
$$

where $\left({ }^{H} D_{1^{+}}^{\alpha} y\right)(\cdot)$ is the Hadamard derivative of order $\alpha \in(0,1), \mathbb{A}, \mathbb{B} \in \mathbb{R}^{n \times n}$ denote constant matrices, and $\varphi:\left[\frac{1}{h}, 1\right] \rightarrow \mathbb{R}^{n}$ is an arbitrary Hadamard differentiable vector function, $f \in C\left([1, T], \mathbb{R}^{n}\right), T=h^{l}$ for a fixed natural number $l$.

The second purpose of this paper is to study the existence and stability of solutions for a Hadamard type fractional delay differential equation

$$
\left\{\begin{array}{c}
\left({ }^{H} D_{1^{+}}^{\alpha} y\right)(t)=\mathbb{A} y(t)+\mathbb{B} y\left(\frac{t}{h}\right)+f(t, y(t)), \quad t \in(1, T], h>0, \\
y(t)=\varphi(t), \frac{1}{h}<t \leq 1, \\
\left({ }^{H} I_{1^{+}}^{1-\alpha} y\right)\left(1^{+}\right)=a \in \mathbb{R}^{n},
\end{array}\right.
$$

At the end of this section, we state the main contribution of the paper as follows:

(i) We propose delayed perturbation of the M-L type functions $Y_{h, \alpha, \beta}^{\mathbb{A}, \mathbb{B}}(t, s)$ with logarithms, by means of the matrix Equations (6). We show that for $\mathbb{B}=\Theta$ the function $Y_{h, \alpha, \beta}^{\mathbb{A}, \mathbb{B}}(t, s)$ coincides with the M-L type function with two parameters $(\ln t-\ln s)^{\beta-1} E_{\alpha, \beta}\left(\mathbb{A}(\ln t-\ln s)^{\alpha}\right)$. For $\mathbb{A}=\Theta$ the delayed M-L type function $Y_{h, \alpha, \beta}^{\mathbb{A}, \mathbb{B}}(t, s)$ coincides with the delayed M-L type matrix function with two parameters $E_{h, \alpha, \beta}^{\mathbb{B}}(\ln t-\ln h)$, introduced in (4).

(ii) We explicitly write the solution of the Hadamard type fractional delay linear system (2) via delayed perturbation of the M-L type function with logarithm. Using this representation we study existence, uniqueness, and Ulam-Hyers stability of the nonlinear Equation (3). 


\section{Preliminaries}

Let $0<a<b<\infty$ and $C[a, b]$ be the Banach space of all continuous functions $y:[a, b] \rightarrow \mathbb{R}^{n}$ with the norm $\|y\|_{C}:=\max \{\|y(t)\|: t \in[a, b]\}$. For $0 \leq \gamma<1$, we denote the space $C_{\gamma, \ln }(a, b]$ by the weighted Banach space of the continuous function $y:[a, b] \rightarrow \mathbb{R}^{n}$, which is given by

$$
C_{\gamma, \ln }(a, b]:=\left\{y(t):\left(\ln \frac{t}{a}\right)^{\gamma} y(t) \in C[a, b]\right\},
$$

endowed with the norm $\|y\|_{\gamma}:=\sup \left\{\left(\ln \frac{t}{a}\right)^{\gamma}\|y(t)\|: t \in(a, b]\right\}$.

The following definitions and lemmas will be used in this paper.

Definition 1. [24] Hadamard fractional integral of order $\alpha \in \mathbb{R}^{+}$of function $y(t)$ is defined by

$$
\left({ }^{H} I_{a^{+}}^{\alpha} y\right)(t)=\frac{1}{\Gamma(\alpha)} \int_{a}^{t}\left(\ln \frac{t}{s}\right)^{\alpha-1} y(s) \frac{d s}{s}, 1 \leq a<t \leq b,
$$

where $\Gamma$ is the Gamma function.

Definition 2. [24] Hadamard fractional derivative of order $\alpha \in[n-1, n), n \in \mathbb{Z}^{+}$of function $y(t)$ is defined by

$$
\left({ }^{H} D_{a^{+}}^{\alpha} y\right)(t)=\frac{1}{\Gamma(n-\alpha)}\left(t \frac{d}{d t}\right)^{n} \int_{a}^{t}\left(\ln \frac{t}{s}\right)^{n-\alpha+1} y(s) \frac{d s}{s}, 1 \leq a<t \leq b .
$$

Lemma 1. [24] If $a, \gamma, \beta>0$ then

- $\quad\left({ }^{H} I_{a^{+}}^{\gamma}\left(\ln \frac{t}{a}\right)^{\beta-1}\right)(t)=\frac{\Gamma(\beta)}{\Gamma(\beta+\gamma)}\left(\ln \frac{t}{a}\right)^{\beta+\gamma-1}$.

- $\left({ }^{H} D_{a^{+}}^{\gamma}\left(\ln \frac{t}{a}\right)^{\beta-1}\right)(t)=\frac{\Gamma(\beta)}{\Gamma(\beta-\gamma)}\left(\ln \frac{t}{a}\right)^{\beta-\gamma-1}$.

- $\quad$ For $0<\beta<1,\left({ }^{H} D_{a^{+}}^{\beta}\left(\ln \frac{t}{a}\right)^{\beta-1}\right)(t)=0$.

Definition 3. M-L type matrix function with two parameters $e_{\alpha, \beta}(\mathbb{A} ; t): \mathbb{R} \rightarrow \mathbb{R}^{n \times n}$ is defined by

$$
e_{\alpha, \beta}(\mathbb{A} ; t):=t^{\beta-1} E_{\alpha, \beta}(\mathbb{A} ; t):=t^{\beta-1} \sum_{k=0}^{\infty} \frac{\mathbb{A}^{k} t^{\alpha k}}{\Gamma(k \alpha+\beta)}, \quad \alpha, \beta>0, t \in \mathbb{R}
$$

Next, we introduce a definition of delayed M-L type matrix function $E_{h, \alpha, \beta}^{\mathbb{B}}(\ln t): \mathbb{R}^{+} \rightarrow \mathbb{R}^{n \times n}$ with logarithm generated by $\mathbb{B}$.

Definition 4. Two parameters delayed $M-L$ type matrix function $E_{h, \alpha, \beta}^{\mathbb{B}}(\ln t): \mathbb{R}^{+} \rightarrow \mathbb{R}^{n \times n}$ with logarithm generated by $\mathbb{B}$ is defined by

$$
E_{h, \alpha, \beta}^{\mathbb{B}}(\ln t):= \begin{cases}\Theta, & 0<t \leq \frac{1}{h} \\ I \frac{(\ln t+\ln h)^{\beta-1}}{\Gamma(\beta)}, & 1 \frac{1}{h}<t \leq 1, \\ I \frac{(\ln t+\ln h)^{\beta-1}}{\Gamma(\beta)}+\mathbb{B} \frac{(\ln t)^{\alpha+\beta-1}}{\Gamma(\alpha+\beta)}+\ldots+\mathbb{B}^{p \frac{(\ln t-(p-1) \ln h)^{p \alpha+\beta-1}}{\Gamma(p \alpha+\beta)},}, & h^{p-1}<t \leq h^{p} .\end{cases}
$$

Our definition of the two-parameter delayed M-L type matrix function with logarithm differs substantially from the definition given in [33]. 
In order to give a definition of delayed perturbation of the M-L type matrix functions with logarithm, we introduce the following matrices $Y_{\alpha, \beta, k}, k=0,1,2, \ldots$

$$
\begin{aligned}
Y_{\alpha, \beta, 0}(t, s) & =\left(\ln \frac{t}{s}\right)^{\beta-1} E_{\alpha, \beta}\left(\mathbb{A} ; \ln \frac{t}{s}\right), \\
Y_{\alpha, \beta, 1}(t, s h) & =\int_{s h}^{t} e_{\alpha, \alpha}\left(\mathbb{A} ; \ln \frac{t}{r}\right) \mathbb{B} Y_{\alpha, \beta, 0}\left(\frac{r}{h}, s\right) \frac{d r}{r}, \\
Y_{\alpha, \beta, k}\left(t, s h^{k}\right) & =\int_{s h^{k}}^{t} e_{\alpha, \alpha}\left(\mathbb{A} ; \ln \frac{t}{r}\right) \mathbb{B} Y_{\alpha, \beta, k-1}\left(\frac{r}{h}, s h^{k-1}\right) \frac{d r}{r} .
\end{aligned}
$$

Definition 5. Let $\mathbb{A}, \mathbb{B} \in \mathbb{R}^{n \times n}$ be fixed matrices and $k \in \mathbb{N} \cup\{0\}$. Delayed perturbation of $M-L$ type function $Y_{h, \alpha, \beta}^{\mathbb{A}, \mathbb{B}}(\cdot, \cdot): \mathbb{R} \times \mathbb{R} \rightarrow \mathbb{R}^{n}$ with logarithm generated by $\mathbb{A}, \mathbb{B}$ is defined by

$$
\begin{aligned}
Y_{h, \alpha, \beta}^{\mathbb{A}, \mathbb{B}}(t, s) & :=\sum_{j=0}^{\infty} Y_{\alpha, \beta, j}\left(t, s h^{j}\right) H\left(t-s h^{j}\right) \\
& = \begin{cases}\Theta, & 0<t<s, \\
I, & t=s, \\
Y_{\alpha, \beta, 0}(t, s)+Y_{\alpha, \beta, 1}(t, s h)+\ldots+Y_{\alpha, \beta, k}\left(t, s h^{k}\right), & s h^{k}<t \leq s h^{k+1},\end{cases}
\end{aligned}
$$

where $H(t)$ is a Heaviside function: $H(t)=\left\{\begin{array}{l}1, t>0 \\ 0, \quad t \leq 0\end{array}\right.$

Lemma 2. Let $a, b>-1$. For $s h^{k}<t \leq s h^{k+1}, k \in \mathbb{N} \cup\{0\}$, one has

$$
\begin{gathered}
\int_{r}^{t}\left(\ln \frac{t}{s}\right)^{a}\left(\ln \frac{s}{r}\right)^{b} \frac{d s}{s}=\left(\ln \frac{t}{r}\right)^{a+b+1} \mathcal{B}[a+1, b+1], \\
\frac{1}{\Gamma(1-\alpha)} \int_{s h^{k}}^{t}\left(\ln \frac{t}{r}\right)^{-\alpha} Y_{\alpha, \beta, k}\left(r, s h^{k}\right) \frac{d r}{r}=\int_{s h^{k}}^{t} E_{\alpha, 1}\left(\mathbb{A} ; \ln \frac{t}{r}\right) \mathbb{B} Y_{\alpha, \beta, k-1}\left(\frac{r}{h}, s h^{k-1}\right) \frac{d r}{r} .
\end{gathered}
$$

Proof. Let $\ln \frac{s}{r}=\tau \ln \frac{t}{r}$. Then $s=r\left(\frac{t}{r}\right)^{\tau}, d s=r\left(\frac{t}{r}\right)^{\tau} \ln \frac{t}{r} d \tau$. So we have

$$
\ln \frac{t}{s}=\ln \left(\frac{t}{r}\right)^{1-\tau}=(1-\tau) \ln \left(\frac{t}{r}\right)
$$

and

$$
\begin{aligned}
\int_{r}^{t}\left(\ln \frac{t}{s}\right)^{a}\left(\ln \frac{s}{r}\right)^{b} \frac{d s}{s} & =\int_{0}^{1}\left(\ln \frac{t}{r}\right)^{a}(1-\tau)^{a}\left(\ln \frac{t}{r}\right)^{b} \tau^{b} \ln \frac{t}{r} d \tau \\
& =\left(\ln \frac{t}{r}\right)^{a+b+1} \mathcal{B}[a+1, b+1] .
\end{aligned}
$$

To prove (8), firstly using (7) we calculate it for $k=0$ :

$$
\begin{aligned}
\frac{1}{\Gamma(1-\alpha)} \int_{s}^{t}\left(\ln \frac{t}{r}\right)^{-\alpha} Y_{\alpha, \beta, 0}(r, s) \frac{d r}{r} & =\frac{1}{\Gamma(1-\alpha)} \frac{1}{\Gamma(\beta)} \int_{s}^{t}\left(\ln \frac{t}{r}\right)^{-\alpha}\left(\ln \frac{r}{s}\right)^{\beta-1} \frac{d r}{r} \\
& =\frac{(\ln t-\ln s)^{-\alpha+\beta}}{\Gamma(-\alpha+\beta+1)}
\end{aligned}
$$


Similarly, for any $k \in \mathbb{N}$, we have

$$
\begin{aligned}
& \frac{1}{\Gamma(1-\alpha)} \int_{s h^{k}}^{t}\left(\ln \frac{t}{r}\right)^{-\alpha} Y_{\alpha, \beta, k}\left(r, s h^{k}\right) \frac{d r}{r} \\
& =\frac{1}{\Gamma(1-\alpha)} \int_{s h^{k}}^{t}\left(\ln \frac{t}{r}\right)^{-\alpha} \int_{s h^{k}}^{r} e_{\alpha, \alpha}\left(\mathbb{A} ; \ln \frac{r}{r_{1}}\right) \mathbb{B} Y_{\alpha, \beta, k-1}\left(\frac{r_{1}}{h}, s h^{k-1}\right) H\left(r-s h^{k}\right) \frac{d r_{1}}{r_{1}} \frac{d r}{r} \\
& =\frac{1}{\Gamma(1-\alpha)} \int_{s h^{k}}^{t} \int_{r_{1}}^{t}\left(\ln \frac{t}{r}\right)^{-\alpha} e_{\alpha, \alpha}\left(\mathbb{A} ; \ln \frac{r}{r_{1}}\right) \frac{d r}{r} \mathbb{B} Y_{\alpha, \beta, k-1}\left(\frac{r_{1}}{h}, s h^{k-1}\right) H\left(r-s h^{k}\right) \frac{d r_{1}}{r_{1}} \\
& =\int_{s h^{k}}^{t} E_{\alpha, 1}\left(\mathbb{A} ; \ln \frac{t}{r_{1}}\right) \mathbb{B} Y_{\alpha, \beta, k-1}\left(\frac{r_{1}}{h}, s h^{k-1}\right) \frac{d r_{1}}{r_{1}} .
\end{aligned}
$$

Lemma 3. If $\mathbb{A}$ and $\mathbb{B}$ are commutative matrices, then

$$
Y_{\alpha, \beta, k}\left(t, s h^{k}\right)=\mathbb{B}^{k} \sum_{n=0}^{\infty}\left(\begin{array}{c}
n+k \\
k
\end{array}\right) \mathbb{A}^{n} \frac{\left(\ln t-\ln s h^{k}\right)^{n \alpha+k \alpha+\beta-1}}{\Gamma(n \alpha+k \alpha+\beta)} .
$$

Proof. The proof is based on the equality (7). Using Lemma 2, for $k=1$, we have

$$
\begin{aligned}
Y_{\alpha, \beta, 1}(t, s h) & =\int_{s h}^{t} e_{\alpha, \alpha}\left(\mathbb{A} ; \ln \frac{t}{r}\right) \mathbb{B} Y_{\alpha, \beta, 0}\left(\frac{r}{h}, s\right) \frac{d r}{r} \\
& =\mathbb{B} \int_{s h}^{t} \sum_{n=0}^{\infty} \mathbb{A}^{n}\left(\ln \frac{t}{r}\right)^{\alpha n+\alpha-1} \frac{1}{\Gamma(\alpha n+\alpha)} \sum_{k=0}^{\infty} \mathbb{A}^{k}\left(\ln \frac{r}{s h}\right)^{\alpha n+\beta-1} \frac{1}{\Gamma(\alpha k+\beta)} \frac{d r}{r} \\
& =\mathbb{B} \int_{s h}^{t} \sum_{n=0}^{\infty} \sum_{k=0}^{k} \mathbb{A}^{k}\left(\ln \frac{t}{r}\right)^{\alpha k+\alpha-1} \frac{1}{\Gamma(\alpha k+\alpha)} \mathbb{A}^{n-k}\left(\ln \frac{r}{s h}\right)^{\alpha(n-k)+\beta-1} \frac{1}{\Gamma(\alpha(n-k)+\beta)} \frac{d r}{r} \\
& =\mathbb{B} \sum_{n=0}^{\infty} \sum_{k=0}^{k} \mathbb{A}^{n} \frac{1}{\Gamma(\alpha k+\alpha) \Gamma(\alpha(n-k)+\beta)} \int_{s h}^{t}\left(\ln \frac{t}{r}\right)^{\alpha k+\alpha-1}\left(\ln \frac{r}{s h}\right)^{\alpha(n-k)+\beta-1} \frac{d r}{r} \\
& =\mathbb{B} \sum_{n=0}^{\infty} \sum_{k=0}^{k} \mathbb{A}^{n} \frac{(\ln t-\ln s h)^{\alpha n+\alpha+\beta-1}}{\Gamma(\alpha k+\alpha) \Gamma(\alpha(n-k)+\beta)} \mathcal{B}(\alpha k+\alpha, \alpha(n-k)+\beta) \\
& =\mathbb{B} \sum_{n=0}^{\infty}\left(\begin{array}{c}
n+1 \\
1
\end{array}\right) \mathbb{A}^{n} \frac{(\ln t-\ln s h)^{\alpha n+\alpha+\beta-1}}{\Gamma(\alpha n+\alpha+\beta)} .
\end{aligned}
$$

For $k=2$, we get

$$
\begin{aligned}
Y_{\alpha, \beta, 2}\left(t, s h^{2}\right)= & \int_{s h^{2}}^{t} e_{\alpha, \alpha}\left(\mathbb{A} ; \ln \frac{t}{r}\right) \mathbb{B} Y_{\alpha, \beta, 1}\left(\frac{r}{h^{\prime}}, s h\right) d r \\
= & \mathbb{B} \int_{s h^{2}}^{t} \sum_{n=0}^{\infty} \sum_{k=0}^{n} \mathbb{A}^{k}\left(\ln \frac{t}{r}\right)^{k \alpha+\alpha-1} \frac{1}{\Gamma(k \alpha+\alpha)} \mathbb{B}\left(\begin{array}{c}
n+1-k \\
1
\end{array}\right) \\
& \times \mathbb{A}^{n-k}\left(\ln \frac{r}{s h^{2}}\right)^{n \alpha-k \alpha+\alpha+\beta-1} \frac{1}{\Gamma(n \alpha-k \alpha+\alpha+\beta)} \\
= & \mathbb{B}^{2} \sum_{n=0}^{\infty} \sum_{k=0}^{n}\left(\begin{array}{c}
n+1-k \\
1
\end{array}\right) \mathbb{A}^{n} \frac{1}{\Gamma(k \alpha+\beta) \Gamma(n \alpha-k \alpha+\alpha+\beta)} \int_{s h^{2}}^{t}\left(\ln \frac{t}{r}\right)^{k \alpha+\alpha-1}\left(\ln \frac{r}{s h^{2}}\right)^{n \alpha-k \alpha+\alpha+\beta-1} d r \\
= & \mathbb{B}^{2} \sum_{n=0}^{\infty}\left(\sum_{k=0}^{n}\left(\begin{array}{c}
n+1-k \\
1
\end{array}\right)\right) \mathbb{A}^{n}\left(\ln \frac{r}{s h^{2}}\right)^{n \alpha+2 \alpha+\beta-1} \frac{1}{\Gamma(n \alpha+2 \alpha+\beta)} \\
= & \mathbb{B}^{2} \sum_{n=0}^{\infty}\left(\begin{array}{c}
n+2 \\
2
\end{array}\right) \mathbb{A}^{n} \frac{\left(\ln t-\ln s h^{2}\right)^{n \alpha+2 \alpha+\beta-1}}{\Gamma(n \alpha+2 \alpha+\beta)} .
\end{aligned}
$$

Using the Mathematical Induction in a similar manner we can get (10). 
According to Lemma 3 in the case $\mathbb{A B}=\mathbb{B} \mathbb{A}$ delayed M-L type function $Y_{h, \alpha, \beta}^{\mathbb{A}, \mathbb{B}}(t, s)$ has a simple form:

$$
Y_{h, \alpha, \beta}^{\mathbb{A}, \mathbb{B}}(t, s):= \begin{cases}\Theta, & 0<t<s, \\
I, & t=s, \\
\sum_{i=0}^{\infty} \mathbb{A}^{i} \frac{(\ln t-\ln s)^{i \alpha+\beta-1}}{\Gamma(i \alpha+\beta)}+\sum_{i=1}^{\infty}\left(\begin{array}{c}
i \\
1
\end{array}\right) \mathbb{A}^{i-1} \mathbb{B} \frac{(\ln t-\ln s h)^{i \alpha+\beta-1}}{\Gamma(i \alpha+\beta)} & \\
+\ldots+\sum_{i=p}^{\infty}\left(\begin{array}{c}
i \\
p
\end{array}\right) \mathbb{A}^{i-p} \mathbb{B}^{p} \frac{\left(\ln t-\ln s h^{p}\right)^{i \alpha+\beta-1}}{\Gamma(i \alpha+\beta)}, & s h^{p}<t \leq s h^{p+1} .\end{cases}
$$

Next lemma shows some special cases of the delayed M-L type function.

Lemma 4. Let $Y_{h, \alpha, \beta}^{\mathbb{A}, \mathbb{B}}(t, s)$ be defined by (6). Then the following holds true:

(i) if $\mathbb{A}=\Theta$ then $Y_{h, \alpha, \beta}^{\mathbb{A}, \mathbb{B}}(t, 1)=E_{h, \alpha, \beta}^{\mathbb{B}}\left(\ln \frac{t}{h}\right), h^{k-1}<\frac{t}{h} \leq h^{k}$,

(ii) if $\mathbb{B}=\Theta$ then $Y_{h, \alpha, \beta}^{\mathbb{A}, \mathbb{B}}(t, s)=\left(\ln \frac{t}{s}\right)^{\beta-1} E_{\alpha, \beta}\left(\mathbb{A}\left(\ln \frac{t}{s}\right)^{\alpha}\right)=e_{\alpha, \beta}\left(\mathbb{A} ; \ln \frac{t}{s}\right)$,

(iii) if $\alpha=\beta=1$ and $\mathbb{A B}=\mathbb{B} \mathbb{A}$ then $Y_{h, 1,1}^{\mathbb{A}, \mathbb{B}}(t, s)=e^{\mathbb{A}(\ln t-\ln s)} e_{h}^{\mathbb{B}(\ln t-\ln h)}, \mathbb{B}_{1}=\mathbb{B} e^{-\mathbb{A} \ln h}$, sh $<t \leq$ $s h^{k+1}$.

Proof. (i) If $\mathbb{A}=\Theta$, then the formula (5)

$$
\begin{aligned}
Y_{\alpha, \beta, 0}(t, s) & =e_{\alpha, \beta}\left(\Theta, \ln \frac{t}{s}\right)=I \frac{(\ln t-\ln s)^{\beta-1}}{\Gamma(\beta)}, \\
Y_{\alpha, \beta, 1}(t, s h) & =\int_{s h}^{t} e_{\alpha, \alpha}\left(\Theta, \ln \frac{t}{r}\right) \mathbb{B} Y_{0}\left(\frac{r}{h}, s\right) \frac{d r}{r}=\frac{1}{\Gamma(\alpha) \Gamma(\beta)} \int_{s h}^{t}\left(\ln \frac{t}{r}\right)^{\alpha-1}\left(\ln \frac{r}{s h}\right)^{\beta-1} \frac{d r}{r} \\
& =\frac{1}{\Gamma(\alpha) \Gamma(\beta)} \mathbb{B}\left(\ln \frac{t}{s h}\right)^{\alpha+\beta-1} \mathcal{B}[\alpha, \beta]=\frac{1}{\Gamma(\alpha+\beta)} \mathbb{B}\left(\ln \frac{t}{s h}\right)^{\alpha+\beta-1}, \\
Y_{\alpha, \beta, 2}\left(t, s h^{2}\right) & =\int_{s h^{2}}^{t} e_{\alpha, \alpha}\left(\Theta, \ln \frac{t}{r}\right) \mathbb{B} Y_{1}\left(\frac{r}{h}, s h\right) d r=\frac{1}{\Gamma(\alpha) \Gamma(\alpha+\beta)} \mathbb{B}^{2} \int_{s h^{2}}^{t}\left(\ln \frac{t}{r}\right)^{\alpha-1}\left(\ln \frac{r}{s h^{2}}\right)^{\alpha+\beta-1} \frac{d r}{r} \\
& =\frac{1}{\Gamma(\alpha) \Gamma(\alpha+\beta)} \mathbb{B}^{2}\left(\ln \frac{t}{s h^{2}}\right)^{2 \alpha+\beta-1} \mathcal{B}[\alpha, \alpha+\beta]=\frac{1}{\Gamma(2 \alpha+\beta)} \mathbb{B}^{2}\left(\ln \frac{t}{s h^{2}}\right)^{2 \alpha+\beta-1}, \\
Y_{\alpha, \beta, k}\left(t, s h^{k}\right) & =\mathbb{B}^{k}\left(\ln \frac{t}{s h^{k}}\right)^{k \alpha+\beta-1} \frac{1}{\Gamma(k \alpha+\beta)}, k \geq 0 .
\end{aligned}
$$

So $Y_{h, \alpha, \beta}^{\mathbb{A}, \mathbb{B}}(t, 1)$ coincides with $E_{h, \alpha, \beta}^{\mathbb{B}}(\ln t-\ln h)$ :

$$
\begin{aligned}
Y_{h, \alpha, \beta}^{\mathbb{A}, \mathbb{B}}(t, 1) & =\sum_{i=0}^{k} \mathbb{B}^{i}\left(\ln \frac{t}{h^{i}}\right)^{i \alpha+\beta-1} \frac{1}{\Gamma(i \alpha+\beta)}=I \frac{(\ln t)^{\beta-1}}{\Gamma(\beta)}+\mathbb{B} \frac{(\ln t-\ln h)^{\alpha+\beta-1}}{\Gamma(\alpha+\beta)}+\ldots+\mathbb{B}^{k} \frac{(\ln t-k \ln h)^{k \alpha+\beta-1}}{\Gamma(k \alpha+\beta)} \\
& =E_{h, \alpha, \beta}^{\mathbb{B}}(\ln t-\ln h), h^{k-1}<\frac{t}{h} \leq h^{k} .
\end{aligned}
$$

(ii) Trivially, from definition of $Y_{h, \alpha, \beta}^{\mathbb{A}, \mathbb{B}}(t, s)$ we have: if $\mathbb{B}=\Theta$, then

$$
Y_{h, \alpha, \beta}^{\mathbb{A}, \mathbb{B}}(t, s)=\left(\ln \frac{t}{s}\right)^{\beta-1} E_{\alpha, \beta}\left(\mathbb{A}\left(\ln \frac{t}{s}\right)^{\alpha}\right) .
$$


(iii) By (11) for the case $\alpha=\beta=1$ and $\mathbb{A B}=\mathbb{B} \mathbb{A}$, we have

$$
\begin{aligned}
Y_{h, 1,1}^{\mathbb{A}, \mathbb{B}}(t, s) & =\sum_{i=0}^{\infty} \mathbb{A}^{i} \frac{(\ln t-\ln s)^{i}}{\Gamma(i+1)}+\sum_{i=0}^{\infty}\left(\begin{array}{c}
i+1 \\
1
\end{array}\right) \mathbb{A}^{i} \mathbb{B} \frac{(\ln t-\ln s h)^{i+1}}{\Gamma(i+1)} \\
& +\ldots+\sum_{i=0}^{\infty}\left(\begin{array}{c}
i+k \\
k
\end{array}\right) \mathbb{A}^{i} \mathbb{B}^{k} \frac{\left(\ln t-\ln s h^{k}\right)^{i+k}}{\Gamma(i+k+1)} \\
& =e^{\mathbb{A}(\ln t-\ln s)}+e^{\mathbb{A}(\ln t-\ln s h)} \mathbb{B}(\ln t-\ln s h)+\ldots+e^{\mathbb{A}\left(\ln t-\ln s h^{k}\right)} \mathbb{B}^{k} \frac{1}{k !}(\ln t-k \ln h)^{k} \\
& =e^{\mathbb{A}(\ln t-\ln s)} e_{h}^{\mathbb{B}_{1}(\ln t-\ln h)} .
\end{aligned}
$$

Lemma 5. For any $s \in \mathbb{R}$ the function $Y_{h, \alpha, \beta}^{\mathbb{A}, \mathbb{B}}(\cdot, s):(1, \infty) \rightarrow \mathbb{R}^{n \times n}$ is continuous.

Proof. The proof is similar to that of [34] and is omitted.

It turns out that $Y_{h, \alpha, \beta}^{\mathbb{A}, \mathbb{B}}(t, s)$ is a delayed perturbation of the Cauchy matrix with logarithm of the homogeneous Equation (2) with $f=0$.

Lemma 6. $Y_{h, \alpha, \beta}^{\mathbb{A}, \mathbb{B}}: \mathbb{R} \times \mathbb{R} \rightarrow \mathbb{R}^{n \times n}$ is a solution of

$$
{ }^{H} D_{1^{+}}^{\alpha} Y_{h, \alpha, \beta}^{\mathbb{A}, \mathbb{B}}(t, s)=\left(\ln \frac{t}{s}\right)^{-\alpha+\beta-1} \frac{1}{\Gamma(-\alpha+\beta)}+\mathbb{A} Y_{h, \alpha, \beta}^{\mathbb{A}, \mathbb{B}}(t, s)+\mathbb{B} Y_{h, \alpha, \beta}^{\mathbb{A}, \mathbb{B}}\left(\frac{t}{h^{\prime}}, s\right) .
$$

Proof. According to (9) we have

$$
\begin{aligned}
\left({ }^{H} D_{1^{+}}^{\alpha} Y_{\alpha, \beta, 0}(t, s)\right)(t) & =\frac{1}{\Gamma(1-\alpha)}\left(t \frac{d}{d t}\right) \int_{1}^{t}\left(\ln \frac{t}{r}\right)^{-\alpha} Y_{\alpha, \beta, 0}(r, s) \frac{d r}{r} \\
& =\frac{1}{\Gamma(1-\alpha)}\left(t \frac{d}{d t}\right) \int_{1}^{t}\left(\ln \frac{t}{r}\right)^{-\alpha} e_{\alpha, \beta}\left(\mathbb{A} ; \ln \frac{r}{s}\right) \frac{d r}{r} \\
& =\left(t \frac{d}{d t}\right) e_{\alpha, 1-\alpha+\beta}\left(\mathbb{A} ; \ln \frac{t}{s}\right) \\
& =\left(\ln \frac{t}{s}\right)^{-\alpha+\beta-1} \frac{1}{\Gamma(-\alpha+\beta)}+\mathbb{A} e_{\alpha, \beta}\left(\mathbb{A} ; \ln \frac{t}{s}\right) \\
& =\left(\ln \frac{t}{s}\right)^{-\alpha+\beta-1} \frac{1}{\Gamma(-\alpha+\beta)}+\mathbb{A} Y_{\alpha, \beta, 0}(t, s) .
\end{aligned}
$$

On the other hand for any $k \in \mathbb{N}$ :

$$
\begin{aligned}
\left({ }^{H} D_{1^{+}}^{\alpha} Y_{\alpha, \beta, k}\left(t, s h^{k}\right)\right)(t) & =\frac{1}{\Gamma(1-\alpha)}\left(t \frac{d}{d t}\right) \int_{1}^{t}\left(\ln \frac{t}{r}\right)^{-\alpha} Y_{\alpha, \beta, k}(r, s) \frac{d r}{r} \\
& =\left(t \frac{d}{d t}\right) \int_{s h^{k}}^{t} E_{\alpha, 1}\left(\mathbb{A} ; \ln \frac{t}{r}\right) \mathbb{B} Y_{\alpha, \beta, k-1}\left(\frac{r}{h^{\prime}} s h^{k-1}\right) \frac{d r}{r} \\
& =\int_{s h^{k}}^{t}\left(t \frac{d}{d t}\right) E_{\alpha, 1}\left(\mathbb{A} ; \ln \frac{t}{r}\right) \mathbb{B} Y_{\alpha, \beta, k-1}\left(\frac{r}{h}, s h^{k-1}\right) \frac{d r}{r} \\
& +\mathbb{B} Y_{\alpha, \beta, k-1}\left(\frac{t}{h}, s h^{k-1}\right) \\
& =\mathbb{A} Y_{\alpha, \beta, k}\left(t, s h^{k}\right)+\mathbb{B} Y_{\alpha, \beta, k-1}\left(\frac{t}{h^{\prime}} s h^{k-1}\right) .
\end{aligned}
$$


From (13) and (14) it follows that for $s h^{k}<t \leq s h^{k+1}$

$$
\begin{aligned}
{ }^{H} D_{1^{+}}^{\alpha} Y_{h, \alpha, \beta}^{\mathbb{A}, \mathbb{B}}(t, s) & ={ }^{H} D_{1^{+}}^{\alpha} Y_{\alpha, \beta, 0}(t, s)+{ }^{H} D_{1^{+}}^{\alpha} Y_{\alpha, \beta, 1}(t, s h)+\ldots+{ }^{H} D_{1^{+}}^{\alpha} Y_{\alpha, \beta, k}\left(t, s h^{k}\right) \\
& =\left(\ln \frac{t}{s}\right)^{-\alpha+\beta-1} \frac{1}{\Gamma(-\alpha+\beta)}+\mathbb{A} Y_{\alpha, \beta, 0}(t, s)+\mathbb{A} Y_{\alpha, \beta, 1}(t, s h)+\mathbb{B} Y_{\alpha, \beta, 0}\left(\frac{t}{h}, s\right) \\
& +\ldots+\mathbb{A} Y_{\alpha, \beta, k}\left(t, s h^{k}\right)+\mathbb{B} Y_{\alpha, \beta, k-1}\left(\frac{t}{h}, s h^{k-1}\right) \\
& =\left(\ln \frac{t}{s}\right)^{-\alpha+\beta-1} \frac{1}{\Gamma(-\alpha+\beta)}+\mathbb{A} Y_{h, \alpha, \beta}^{\mathbb{A}, \mathbb{B}}(t, s)+\mathbb{B} Y_{h, \alpha, \beta}^{\mathbb{A}, \mathbb{B}}\left(\frac{t}{h}, s\right) .
\end{aligned}
$$

The proof is complete.

Theorem 1. The solution $y(t)$ of (2) with zero initial condition has the form

$$
y(t)=\int_{1}^{t} Y_{h, \alpha, \alpha}^{\mathbb{A}, \mathbb{B}}(t, s) f(s) \frac{d s}{s}, t \geq 0
$$

Proof. Assume that any solution of a nonhomogeneous system $y(t)$ has the form

$$
y(t)=\int_{1}^{t} Y_{h, \alpha, \alpha}^{\mathbb{A}, \mathbb{B}}(t, s) h(s) \frac{d s}{s}, t \geq 0,
$$

where $h(s), 1 \leq s \leq t \leq T$ is an unknown continuous vector function and $y(1)=0$. Having Hadamard fractional differentiation on both sides of (15), for $1<t \leq h$ we have

$$
\begin{aligned}
\left({ }^{H} D_{1}^{\alpha} y\right)(t) & =\mathbb{A} y(t)+\mathbb{B} y\left(\frac{t}{h}\right)+f(t) \\
& =\mathbb{A} \int_{1}^{t} Y_{h, \alpha, \alpha}^{\mathbb{A}, \mathbb{B}}(t, s) h(s) \frac{d s}{s}+\mathbb{B} \int_{1}^{t / h} Y_{h, \alpha, \alpha}^{\mathbb{A}, \mathbb{B}}\left(\frac{t}{h}, s\right) h(s) \frac{d s}{s}+f(t) \\
& =\mathbb{A} \int_{1}^{t} Y_{h, \alpha, \alpha}^{\mathbb{A}, \mathbb{B}}(t, s) h(s) \frac{d s}{s}+f(t) .
\end{aligned}
$$

On the other hand, according to Lemma 2, we have

$$
\begin{aligned}
\left({ }^{H} D_{1+y}^{\alpha}\right)(t) & =\frac{1}{\Gamma(1-\alpha)}\left(t \frac{d}{d t}\right) \int_{1}^{t}\left(\ln \frac{t}{r}\right)^{-\alpha}\left(\int_{1}^{\mathbb{R}} Y_{h, \alpha, \alpha}^{\mathbb{A}, \mathbb{B}}(r, s) h(s) \frac{d s}{s}\right) \frac{d r}{r} \\
& =\frac{1}{\Gamma(1-\alpha)}\left(t \frac{d}{d t}\right) \int_{1}^{t} \int_{s}^{t}\left(\ln \frac{t}{r}\right)^{-\alpha} Y_{h, \alpha, \alpha}^{\mathbb{A}, \mathbb{B}}(r, s) h(s) \frac{d r}{r} \frac{d s}{s} \\
& =c(t)+\frac{1}{\Gamma(1-\alpha)} \int_{1}^{t}\left(t \frac{d}{d t}\right) \int_{s}^{t}\left(\ln \frac{t}{r}\right)^{-\alpha} Y_{h, \alpha, \alpha}^{\mathbb{A}, \mathbb{B}}(r, s) h(s) \frac{d r}{r} \frac{d s}{s} \\
& =h(t)+\mathbb{A} \int_{1}^{t} Y_{h, \alpha, \alpha}^{\mathbb{A}, \mathbb{B}}(t, s) h(s) \frac{d s}{s} .
\end{aligned}
$$

Therefore, $h(t) \equiv f(t)$. The proof is complete.

Theorem 2. The solution $y \in C\left([1, T], \mathbb{R}^{n}\right)$ of $(2)$ with $f=0$ has a form

$$
y(t)=Y_{h, \alpha, \alpha}^{\mathbb{A}, \mathbb{B}}(t, 1) a+\int_{1}^{h} Y_{h, \alpha, \alpha}^{\mathbb{A}, \mathbb{B}}(t, s) \mathbb{B} \varphi\left(\frac{s}{h}\right) \frac{d s}{s} .
$$


Proof. We are looking for a solution which depends on an unknown constant $c$, and a vector function $g(t)$, of the form

$$
y(t)=Y_{h, \alpha, \alpha}^{\mathbb{A}, \mathbb{B}}(t, 1) c+\int_{1}^{h} Y_{h, \alpha, \alpha}^{\mathbb{A}, \mathbb{B}}(t, s) g(s) \frac{d s}{s},
$$

Moreover, $y(t)$ satisfies initial conditions

$$
\begin{aligned}
y(t) & =Y_{h, \alpha, \alpha}^{\mathbb{A}, \mathbb{B}}(t, 1) c+\int_{1}^{h} Y_{h, \alpha, \alpha}^{\mathbb{A}, \mathbb{B}}(t, s) g(s) \frac{d s}{s}, 1<t \leq h, \\
\left({ }^{H} I_{1^{+}}^{1-\alpha} y\right)\left(1^{+}\right) & =a .
\end{aligned}
$$

We have

$$
\begin{aligned}
a & =\left({ }^{H} I_{1^{+}}^{1-\alpha} y\right)\left(1^{+}\right)=\lim _{t \rightarrow 1^{+}}\left({ }^{H} I_{1^{+}}^{1-\alpha} y\right)(t) \\
& =\lim _{t \rightarrow 1^{+}}\left(\frac{1}{\Gamma(1-\alpha)} \int_{1}^{t}(\ln t-\ln s)^{-\alpha} Y_{h, \alpha, \alpha}^{\mathbb{A}, \mathbb{B}}(s, 1) c \frac{d s}{s}\right) \\
& =\lim _{t \rightarrow 1^{+}}\left(\frac{1}{\Gamma(1-\alpha)} \int_{1}^{t}(\ln t-\ln s)^{-\alpha} e_{\alpha, \alpha}(\mathbb{A}, \ln s) \frac{d s}{s} c\right)=c
\end{aligned}
$$

Thus $c=a$. Since $1<t \leq h$, we obtain that

$$
Y_{h, \alpha, \alpha}^{\mathbb{A}, \mathbb{B}}(t, s)= \begin{cases}\left(\ln \frac{t}{s}\right)^{\alpha-1} E_{\alpha, \alpha}\left(\mathbb{A}\left(\ln \frac{t}{s}\right)^{\alpha}\right), & 1 \leq s<t \leq h, \\ \Theta, & t<s \leq h .\end{cases}
$$

Consequently, on interval $1<t \leq h$, we can easily derive

$$
\begin{aligned}
y(t) & =Y_{h, \alpha, \alpha}^{\mathbb{A}, \mathbb{B}}(t, 1) a+\int_{1}^{h} Y_{h, \alpha, \alpha}^{\mathbb{A}, \mathbb{B}}(t, s) g(s) \frac{d s}{s} \\
& =Y_{h, \alpha, \alpha}^{\mathbb{A}, \mathbb{B}}(t, 1) a+\int_{1}^{t} Y_{h, \alpha, \alpha}^{\mathbb{A}, \mathbb{B}}(t, s) g(s) \frac{d s}{s}+\int_{t}^{h} Y_{h, \alpha, \alpha}^{\mathbb{A}, \mathbb{B}}(t, s) g(s) \frac{d s}{s} \\
& =(\ln t)^{\alpha-1} E_{\alpha, \alpha}\left(\mathbb{A}(\ln t)^{\alpha}\right) a+\int_{1}^{t}\left(\ln \frac{t}{s}\right)^{\alpha-1} E_{\alpha, \alpha}\left(\mathbb{A}\left(\ln \frac{t}{s}\right)^{\alpha}\right) g(s) \frac{d s}{s} .
\end{aligned}
$$

Having differentiated (16) in the Hadamard sense, we obtain

$$
\begin{aligned}
\left({ }^{H} D_{1}^{\alpha} y\right)(t) & =\mathbb{A}(\ln t)^{\alpha-1} E_{\alpha, \alpha}\left(\mathbb{A}(\ln t)^{\alpha}\right) a+\mathbb{A} \int_{1}^{t}\left(\ln \frac{t}{s}\right)^{\alpha-1} E_{\alpha, \alpha}\left(\mathbb{A}\left(\ln \frac{t}{s}\right)^{\alpha}\right) g(s) \frac{d s}{s}+g(t) \\
& =\mathbb{A} y(t)+g(t) .
\end{aligned}
$$

Therefore, $g(t)=\left({ }^{H} D_{1^{+}}^{\alpha} y\right)(t)-\mathbb{A} y(t)=\mathbb{B} \varphi\left(\frac{t}{h}\right)$ and the desired formula holds.

Combining Theorems 1 and 2 together we get the following result.

Corollary 1. A solution $y \in C\left([1, T], \mathbb{R}^{n}\right)$ of (2) has a form

$$
\begin{aligned}
y(t) & =Y_{h, \alpha, \alpha}^{\mathbb{A}, \mathbb{B}}(t, 1) a+\int_{1}^{h} Y_{h, \alpha, \alpha}^{\mathbb{A}, \mathbb{B}}(t, s) \mathbb{B} \varphi\left(\frac{s}{h}\right) \frac{d s}{s} \\
& +\int_{1}^{t} Y_{h, \alpha, \alpha}^{\mathbb{A}, \mathbb{B}}(t, s) f(s) \frac{d s}{s} .
\end{aligned}
$$




\section{Existence Uniqueness and Stability}

In this section, we consider the following equivalent integral form of the nonlinear Cauchy problem for fractional time-delay differential equations with Hadamard derivative (3):

$$
\begin{aligned}
y(t) & =Y_{h, \alpha, \alpha}^{\mathbb{A}, \mathbb{B}}(t, 1) a+\int_{1}^{h} Y_{h, \alpha, \alpha}^{\mathbb{A}, \mathbb{B}}(t, s) \mathbb{B} \varphi\left(\frac{s}{h}\right) \frac{d s}{s} \\
& +\int_{1}^{t} Y_{h, \alpha, \alpha}^{\mathbb{A}, \mathbb{B}}(t, s) f(s, y(s)) \frac{d s}{s} .
\end{aligned}
$$

Let us introduce the conditions under which existence and uniqueness of the integral Equation (17) will be investigated.

(A1) $\quad f:[1, T] \times \mathbb{R}^{n} \rightarrow \mathbb{R}^{n}$ be a function such that $f(t, y) \in C_{\gamma, \ln }[1, T]$ with $\gamma<\alpha$ for any $y \in \mathbb{R}^{n}$;

(A2) There exists a positive constant $L_{f}>0$ such that

$$
\left\|f\left(t, y_{1}\right)-f\left(t, y_{2}\right)\right\| \leq L_{f}\left\|y_{1}-y_{2}\right\|,
$$

for each $\left(t, y_{1}\right),\left(t, y_{2}\right) \in[1, T] \times \mathbb{R}^{n}$.

From (A1) and (A2), it follows that

$$
\|f(t, y)\| \leq L_{f}\|y\|+L_{2} \text { for some } L_{2}>0 .
$$

To prove existence uniqueness and stability of (17) we use the following properties of $Y_{\alpha, \beta}^{\mathbb{A}, \mathbb{B}}(t, s)$.

Lemma 7. We have for $\operatorname{sh}^{p}<t \leq \operatorname{sh}^{p+1}, p=0,1, \ldots$,

$$
\left\|Y_{h, \alpha, \beta}^{\mathbb{A}, \mathbb{B}}(t, s)(t, s)\right\| \leq Y_{h, \alpha, \beta}^{\|\mathbb{A}\|,\|\mathbb{B}\|}(t, s) \leq Y_{1, \alpha, \beta}^{\|\mathbb{A}\|,\|\mathbb{B}\|}(t, 1) .
$$

Proof. Indeed,

$$
\begin{aligned}
\left\|Y_{h, \alpha, \beta}^{\mathbb{A}, \mathbb{B}}(t, s)\right\| & \leq Y_{h, \alpha, \beta}^{\|\mathbb{A}\|,\|\mathbb{B}\|}(t, s)=\sum_{k=0}^{p} \sum_{n=0}^{\infty}\left(\begin{array}{c}
n+k \\
k
\end{array}\right)\|\mathbb{B}\|^{k}\|\mathbb{A}\|^{n} \frac{\left(\ln t-\ln s h^{k}\right)^{n \alpha+k \alpha+\beta-1}}{\Gamma(n \alpha+k \alpha+\beta)} \\
& \leq \sum_{k=0}^{p} \sum_{n=0}^{\infty}\left(\begin{array}{c}
n+k \\
k
\end{array}\right)\|\mathbb{B}\|^{k}\|\mathbb{A}\|^{n} \frac{(\ln t)^{n \alpha+k \alpha+\beta-1}}{\Gamma(n \alpha+k \alpha+\beta)} \\
& =\sum_{k=0}^{p} \sum_{n=0}^{\infty}\left(\begin{array}{c}
n+k \\
k
\end{array}\right)\|\mathbb{B}\|^{k}\|\mathbb{A}\|^{n} \frac{(\ln t)^{n \alpha+k \alpha+\beta-1}}{\Gamma(n \alpha+k \alpha+\beta)} \\
& =Y_{1, \alpha, \beta}^{\|\mathbb{A}\|,\|\mathbb{B}\|}(t, 1) .
\end{aligned}
$$

Our first result on existence and uniqueness of (17) is based on the Banach contraction principle.

Theorem 3. Assume that (A1), (A2) hold. If

$$
L_{f}(\ln T) Y_{h, \alpha, \alpha}^{\|\mathbb{A}\|,\|\mathbb{B}\|}(T, 1)<1,
$$

then the Cauchy problem (3) has a unique solution on $[1, T]$. 
Proof. We define an operator $\Theta$ on $\mathcal{B}_{r}:=\left\{y \in C_{\gamma, \ln }[1, T]:\|y\|_{\gamma} \leq r\right\}$ as follows

$$
\begin{aligned}
(\Theta y)(t) & =Y_{h, \alpha, \alpha}^{\mathbb{A}, \mathbb{B}}(t, 1) a+\int_{1}^{h} Y_{h, \alpha, \alpha}^{\mathbb{A}, \mathbb{B}}(t, s) \mathbb{B} \varphi\left(\frac{s}{h}\right) \frac{d s}{s} \\
& +\int_{1}^{t} Y_{h, \alpha, \alpha}^{\mathbb{A}, \mathbb{B}}(t, s) f(s, y(s)) \frac{d s}{s},
\end{aligned}
$$

where $r \geq \frac{M_{2}}{1-M_{1}}$

$$
\begin{aligned}
M_{2}: & =(\ln t)^{\gamma} Y_{1, \alpha, \alpha}^{\|\mathbb{A}\|,\|\mathbb{B}\|}(t, 1)\|a\|+(\ln h)^{-\gamma+1}(\ln t)^{\gamma} Y_{h, \alpha, \alpha}^{\|\mathbb{A}\|,\|\mathbb{B}\|}(T, 1)\left\|\mathbb{B} \varphi\left(\frac{s}{h}\right)\right\|_{\gamma, \ln } \\
& +L_{2}(\ln t)^{\gamma+1} Y_{1, \alpha, \alpha}^{\|\mathbb{A}\|,\|\mathbb{B}\|}(T, 1), \\
M_{1}: & =L_{f}(\ln t)^{\gamma} Y_{h, \alpha, \alpha}^{\|\mathbb{A}\|,\|\mathbb{B}\|}(T, 1)\|y\|_{\gamma, \ln } .
\end{aligned}
$$

It is obvious that $\Theta$ is well-defined due to (A1). Therefore, the existence of a solution of the Cauchy problem (3) is equivalent to that of the operator $\Theta$ has a fixed point on $\mathcal{B}_{r}$. We will use the Banach contraction principle to prove that $\Theta$ has a fixed point. The proof is divided into two steps.

Step 1. $\Theta y \in \mathcal{B}_{r}$ for any $y \in \mathcal{B}_{r}$.

Indeed, for any $y \in \mathcal{B}_{r}$ and any $\delta>0$, by (A3)

$$
\begin{aligned}
\left\|(\ln t)^{\gamma}(\Theta y)(t)\right\| & \leq(\ln t)^{\gamma} Y_{1, \alpha, \alpha}^{\|\mathbb{A}\|,\|\mathbb{B}\|}(t, 1)\|a\|+(\ln t)^{\gamma} \int_{1}^{h} Y_{h, \alpha, \alpha}^{\|\mathbb{A}\|,\|\mathbb{B}\|}(t, s)\left\|\mathbb{B} \varphi\left(\frac{s}{h}\right)\right\| \frac{d s}{s} \\
& +(\ln t)^{\gamma} \int_{1}^{t} Y_{h, \alpha, \alpha}^{\|\mathbb{A}\|,\|\mathbb{B}\|}(t, s)\|f(s, y(s))\| \frac{d s}{s}
\end{aligned}
$$

Firstly, we estimate the first integral:

$$
\begin{aligned}
& (\ln t)^{\gamma} \int_{1}^{h} Y_{h, \alpha, \alpha}^{\|\mathbb{A}\|,\|\mathbb{B}\|}(t, s)\left\|\mathbb{B} \varphi\left(\frac{s}{h}\right)\right\| \frac{d s}{s} \\
& \leq(\ln t)^{\gamma} Y_{h, \alpha, \alpha}^{\|\mathbb{A}\|,\|\mathbb{B}\|}(T, 1) \int_{1}^{h}(\ln s)^{-\gamma} \frac{d s}{s}\left\|\mathbb{B} \varphi\left(\frac{s}{h}\right)\right\|_{\gamma, \ln } \\
& \leq(\ln t)^{\gamma} Y_{h, \alpha, \alpha}^{\|\mathbb{A}\|,\|\mathbb{B}\|}(T, 1)(\ln h)^{-\gamma+1}\left\|\mathbb{B} \varphi\left(\frac{s}{h}\right)\right\|_{\gamma, \ln } \\
& =(\ln h)^{-\gamma+1}(\ln t)^{\gamma} Y_{h, \alpha, \alpha}^{\|\mathbb{A}\|,\|\mathbb{B}\|}(T, 1)\left\|\mathbb{B} \varphi\left(\frac{s}{h}\right)\right\|_{\gamma, \ln } .
\end{aligned}
$$

Similarly,

$$
\begin{aligned}
& (\ln t)^{\gamma} \int_{1}^{t} Y_{h, \alpha, \alpha}^{\|\mathbb{A}\|,\|\mathbb{B}\|}(t, s)\|f(s, y(s))\| \frac{d s}{s} \\
& \leq(\ln t)^{\gamma} \int_{1}^{t} Y_{h, \alpha, \alpha}^{\|\mathbb{A}\|,\|\mathbb{B}\|}(t, s)\left(L_{1}\|y(s)\|+L_{2}\right) \frac{d s}{s} \\
& \leq L_{f}(\ln t)^{\gamma} Y_{h, \alpha, \alpha}^{\|\mathbb{A}\|,\|\mathbb{B}\|}(T, 1)\|y\|_{\gamma, \ln }+L_{2}(\ln t)^{\gamma+1} Y_{1, \alpha, \alpha}^{\|\mathbb{A}\|,\|\mathbb{B}\|}(T, 1) .
\end{aligned}
$$

Inserting (19) and (20) into (18) we get

$$
\begin{aligned}
& \left\|(\ln t)^{\gamma}(\Theta y)(t)\right\| \\
& \leq(\ln t)^{\gamma} Y_{1, \alpha, \alpha}^{\|\mathbb{A}\|,\|\mathbb{B}\|}(t, 1)\|a\|+(\ln h)^{-\gamma+1}(\ln t)^{\gamma} Y_{h, \alpha, \alpha}^{\|\mathbb{A}\|,\|\mathbb{B}\|}(T, 1)\left\|\mathbb{B} \varphi\left(\frac{s}{h}\right)\right\|_{\gamma, \ln } \\
& +L_{f}(\ln t)^{\gamma} Y_{h, \alpha, \alpha}^{\|\mathbb{A}\|,\|\mathbb{B}\|}(T, 1)\|y\|_{\gamma, \ln }+L_{2}(\ln t)^{\gamma+1} Y_{1, \alpha, \alpha}^{\|\mathbb{A}\|,\|\mathbb{B}\|}(T, 1) \\
& \leq M_{2}+M_{1}\|y\|_{\gamma, \ln } \leq M_{2}+M_{1} r \leq r .
\end{aligned}
$$


Step 2. Let $y, z \in C_{\gamma, \ln }[1, T]$. Then similar to the estimation (20) we get

$$
\begin{aligned}
& \left\|(\ln t)^{\gamma}((\Theta y)(t)-(\Theta z)(t))\right\| \leq(\ln t)^{\gamma} \int_{1}^{t} Y_{h, \alpha, \alpha}^{\|\mathbb{A}\|,\|\mathbb{B}\|}(t, s)\|f(s, y(s))-f(s, z(s))\| \frac{d s}{s} \\
& \leq L_{f}(\ln t)^{\gamma} Y_{h, \alpha, \alpha}^{\|\mathbb{A}\|,\|\mathbb{B}\|}(T, 1) \int_{1}^{t}(\ln s)^{-\gamma}(\ln s)^{\gamma}\|y(s)-z(s)\| \frac{d s}{s} \\
& \leq L_{f}(\ln t)^{\gamma} Y_{h, \alpha, \alpha}^{\|\mathbb{A}\|,\|\mathbb{B}\|}(T, 1) \int_{1}^{t}(\ln s)^{-\gamma} \frac{d s}{s}\|y-z\|_{\gamma, \ln } \\
& \leq L_{f} \ln t Y_{h, \alpha, \alpha}^{\|\mathbb{A}\|,\|\mathbb{B}\|}(T, 1)\|y-z\|_{\gamma, \ln }
\end{aligned}
$$

which implies that

$$
\|\Theta y-\Theta z\|_{\gamma, \ln } \leq L_{f}(\ln T) Y_{h, \alpha, \alpha}^{\|\mathbb{A}\|,\|\mathbb{B}\|}(T, 1)\|y-z\|_{\gamma, \ln } .
$$

Hence, the operator $\Theta$ is contraction on $\mathcal{B}_{r}$ and the proof is competed by using the Banach fixed point theorem.

Secondly, we discuss the Ulam-Hyers stability for the problems (3) by means of integral operator given by

$$
y(t)=(\Theta y)(t)
$$

where $\Theta$ is defined by (17).

Define the following nonlinear operator $Q: C_{\gamma, \ln }\left([1, T], \mathbb{R}^{n}\right) \rightarrow C_{\gamma, \ln }\left([1, T], \mathbb{R}^{n}\right)$ :

$$
Q(y)(t):=\left({ }^{H} D_{1^{+}}^{\alpha} y\right)(t)-\mathbb{A} y(t)-\mathbb{B} y\left(\frac{t}{h}\right)-f(t, y(t)) .
$$

For some $\varepsilon>0$, we look at the following inequality:

$$
\|Q(y)\|_{\gamma, \ln } \leq \varepsilon .
$$

Definition 6. We say that the Equation (17) is Ulam-Hyers stable, if there exist $V>0$ such that for every solution $y^{*} \in C_{\gamma, \ln }\left(\left[\frac{1}{h}, T\right], \mathbb{R}^{n}\right)$ of the inequality (22), there exists a unique solution $y \in C_{\gamma, \ln }\left(\left[\frac{1}{h}, T\right], \mathbb{R}^{n}\right)$ of problem (17) with

$$
\left\|y-y^{*}\right\|_{\gamma, \ln } \leq V \varepsilon
$$

Theorem 4. Under the assumptions of Theorem 3, the problem (17) is stable in Ulam-Hyers sense.

Proof. Let $y \in C_{\gamma, \ln }\left(\left[\frac{1}{h}, T\right], \mathbb{R}^{n}\right)$ be the solution of the problem (17). Let $y^{*}$ be any solution satisfying (22):

$$
\left({ }^{H} D_{1+}^{\alpha} y^{*}\right)(t)=\mathbb{A} y^{*}(t)+\mathbb{B} y^{*}\left(\frac{t}{h}\right)+f\left(t, y^{*}(t)\right)+Q\left(y^{*}\right)(t)
$$

So

$$
y^{*}(t)=\Theta\left(y^{*}\right)(t)+\int_{1}^{t} Y_{h, \alpha, \alpha}^{\mathbb{A}, \mathbb{B}}(t, s) Q\left(y^{*}\right)(s) \frac{d s}{s} .
$$

It follows that

$$
\begin{aligned}
& (\ln t)^{\gamma}\left\|\Theta\left(y^{*}\right)(t)-y^{*}(t)\right\| \leq(\ln t)^{\gamma} \int_{1}^{t}\left\|Y_{h, \alpha, \alpha}^{\mathbb{A}, \mathbb{B}}(t, s)\right\|\left\|Q\left(y^{*}\right)(s)\right\| \frac{d s}{s} \\
& \leq(\ln T)^{\gamma+1} Y_{1, \alpha, \alpha}^{\|\mathbb{A}\|,\|\mathbb{B}\|}(T, 1) \varepsilon .
\end{aligned}
$$


Therefore, we deduce by the fixed-point property (21) of the operator $\Theta$, that

$$
\begin{aligned}
(\ln t)^{\gamma}\left\|y(t)-y^{*}(t)\right\| & \leq(\ln t)^{\gamma}\left\|\Theta(y)(t)-\Theta\left(y^{*}\right)(t)\right\|+(\ln t)^{\gamma}\left\|\Theta\left(y^{*}\right)(t)-y^{*}(t)\right\| \\
& \leq L_{f}(\ln T) Y_{h, \alpha, \alpha}^{\|\mathbb{A}\|,\|\mathbb{B}\|}(T, 1)\left\|y-y^{*}\right\|_{\gamma, \ln }+(\ln T)^{\gamma+1} Y_{1, \alpha, \alpha}^{\|\mathbb{A}\|,\|\mathbb{B}\|}(T, 1) \varepsilon,
\end{aligned}
$$

and

$$
\left\|y-y^{*}\right\|_{\gamma, \ln } \leq \frac{(\ln T)^{\gamma+1} Y_{1, \alpha, \alpha}^{\|\mathbb{A}\|,\|\mathbb{B}\|}(T, 1)}{1-L_{f}(\ln T) Y_{h, \alpha, \alpha}^{\|\mathbb{A}\|,\|\mathbb{B}\|}(T, 1)} \varepsilon .
$$

Thus, the problem (3) is Ulam-Hyers stable with

$$
V=\frac{(\ln T)^{\gamma+1} Y_{1, \alpha, \alpha}^{\|\mathbb{A}\|,\|\mathbb{B}\|}(T, 1)}{1-L_{f}(\ln T) Y_{h, \alpha, \alpha}^{\|\mathbb{A}\|,\|\mathbb{B}\|}(T, 1)} .
$$

\section{Existence Result}

Our second existence result is based on the well known Schaefer's fixed point theorem. We use the following linear growth condition to replace $\left(\mathrm{A}_{2}\right)$ :

$\left(\mathrm{A}_{3}\right)$ There exists $M_{f}>0$ such that

$$
\begin{aligned}
\|f(t, y)\| & \leq M_{f}\|y\|, \quad \text { for each } t \in[1, T], y \in \mathbb{R}^{n}, \\
M_{f}(\ln T)^{\gamma} Y_{h, \alpha, \alpha}^{\|\mathbb{A}\|,\|\mathbb{B}\|}(T, 1) & <1 .
\end{aligned}
$$

Theorem 5. Assume that $\left(A_{1}\right)$ and $\left(A_{3}\right)$ hold. Then the Cauchy problem (3) has at least one solution on $C_{\gamma, \ln }[1, T]$.

Proof. Consider the operator $\Theta: C_{\gamma, \ln }[1, T] \rightarrow C_{\gamma, \ln }[1, T]$ defined as follows

$$
\begin{aligned}
(\Theta y)(t) & =Y_{h, \alpha, \alpha}^{\mathbb{A}, \mathbb{B}}(t, 1) a+\int_{1}^{h} Y_{h, \alpha, \alpha}^{\mathbb{A}, \mathbb{B}}(t, s) \mathbb{A} \varphi\left(\frac{s}{h}\right) \frac{d s}{s} \\
& +\int_{1}^{t} Y_{h, \alpha, \alpha}^{\mathbb{A}, \mathbb{B}}(t, s) f(s, y(s)) \frac{d s}{s} .
\end{aligned}
$$

For the sake of convenience, we will split the proof into several steps.

Step 1. $\Theta$ is continuous.

Let $\left\{y_{n}\right\} \subset C_{\gamma, \ln }[1, T]$ be a sequence converging to $y \in C_{\gamma, \ln }[1, T]$. Then for each $t \in[1, T]$, we have

$$
\begin{aligned}
& \left\|(\ln t)^{\gamma}\left(\Theta y_{n}\right)(t)-(\Theta y)(t)\right\| \\
& \leq(\ln t)^{\gamma} \int_{1}^{t}\left\|Y_{h, \alpha, \alpha}^{\mathbb{A}, \mathbb{B}}(t, s)\right\|\left\|f\left(s, y_{n}(s)\right)-f(s, y(s))\right\| \frac{d s}{s} \\
& \leq L_{f}(\ln T) Y_{h, \alpha, \alpha}^{\|\mathbb{A}\|,\|\mathbb{B}\|}(T, 1)\left\|f\left(\cdot, y_{n}(\cdot)\right)-f(\cdot, y(\cdot))\right\|_{\gamma, \ln } .
\end{aligned}
$$

Since $f \in C_{\gamma, \ln }[1, T]$, we have

$$
\begin{aligned}
& \left\|\Theta y_{n}-\Theta y\right\|_{\gamma, \ln } \\
& \leq L_{f}(\ln T) Y_{h, \alpha, \alpha}^{\|\mathbb{A}\|,\|\mathbb{B}\|}(T, 1)\left\|f\left(\cdot, y_{n}(\cdot)\right)-f(\cdot, y(\cdot))\right\|_{\gamma, \ln } \rightarrow 0
\end{aligned}
$$

as $n \rightarrow \infty$.

Step 2. $\Theta$ maps bounded sets into bounded sets in $C_{\gamma, \ln }[1, T]$. 
Step 3. $\Theta$ is equicontinuous of $C_{\gamma, \ln }[1, T]$.

Let $1<t_{1}<t_{2} \leq T, y \in \mathcal{B}_{r}$. Using the assumption $\left(\mathrm{A}_{3}\right)$, we have

$$
\begin{aligned}
& \left\|(\Theta y)\left(t_{2}\right)-(\Theta y)\left(t_{1}\right)\right\| \\
& \leq\left\|Y_{h, \alpha, \alpha}^{\mathbb{A}, \mathbb{B}}\left(t_{2}, 1\right) a-Y_{h, \alpha, \alpha}^{\mathbb{A}, \mathbb{B}}\left(t_{1}, 1\right) a\right\| \\
& +\int_{1}^{h}\left\|\left(Y_{h, \alpha, \alpha}^{\mathbb{A}, \mathbb{B}}\left(t_{2}, s\right)-Y_{h, \alpha, \alpha}^{\mathbb{A}, \mathbb{B}}\left(t_{1}, s\right)\right)\right\|\left\|\mathbb{B} \varphi\left(\frac{s}{h}\right)\right\| \frac{d s}{s} \\
& +\int_{1}^{t_{1}}\left\|\left(Y_{h, \alpha, \alpha}^{\mathbb{A}, \mathbb{B}}\left(t_{1}, s\right)-Y_{h, \alpha, \alpha}^{\mathbb{A}, \mathbb{B}}\left(t_{2}, s\right)\right)\right\|\|f(s, y(s))\| \frac{d s}{s} \\
& +\int_{t_{1}}^{t_{2}}\left\|Y_{h, \alpha, \alpha}^{\mathbb{A}, \mathbb{B}}\left(t_{2}, s\right)\right\|\|f(s, y(s))\| \frac{d s}{s} .
\end{aligned}
$$

The case $1=t_{1}<t_{2} \leq T$ is similar. By Lemmas 5 and 7 , as $t_{2} \rightarrow t_{1}$, the right hand side tends to zero, so $\Theta$ is equicontinuous.

Steps 1-3 imply that $\Theta$ is continuous and completely continuous.

Step 4. A priori bounds.

Now it remains to show that the set

$$
W=\left\{y \in C_{\gamma, \ln }[1, T]: y=\lambda \Theta y, \text { for some } 0<\lambda<1\right\}
$$

is bounded. Assume that $y \in W$, then $y=\lambda \Theta y$ for some $0<\lambda<1$. Thus, for any $t \in[1, T]$, we have

$$
\begin{aligned}
& \left\|(\ln t)^{\gamma} y(t)\right\|=\lambda\left\|(\ln t)^{\gamma}(\Theta y)(t)\right\| \\
& \leq(\ln t)^{\gamma} Y_{1, \alpha, \alpha}^{\|\mathbb{A}\|,\|\mathbb{B}\|}(t, 1)\|a\|+(\ln h)^{-\gamma+1}(\ln t)^{\gamma} Y_{h, \alpha, \alpha}^{\|\mathbb{A}\|,\|\mathbb{B}\|}(T, 1)\left\|\mathbb{B} \varphi\left(\frac{s}{h}\right)\right\|_{\gamma, \ln } \\
& +M_{f}(\ln t)^{\gamma} Y_{h, \alpha, \alpha}^{\|\mathbb{A}\|,\|\mathbb{B}\|}(T, 1)\|y\|_{\gamma, \ln }
\end{aligned}
$$

Since $M_{f}(\ln T)^{\gamma} Y_{h, \alpha, \alpha}^{\|\mathbb{A}\|,\|\mathbb{B}\|}(T, 1)<1$, this shows that the set $W$ is bounded. As a consequence of Schaefer's fixed point theorem, we deduce that $\Theta$ has a fixed point which is a solution of the Cauchy problem (3).

\section{Example}

In this section, we give an examples to illustrate the obtained theoretical result.

Example 1. Let $\alpha=0.3, h=1.2, k=4$. Consider

$$
\begin{aligned}
\left({ }^{H} D_{1^{+}}^{0.3} y\right)(t) & =\mathbb{B} y\left(\frac{t}{1.2}\right), t \in(1,2.0736], \\
y(t) & =\varphi(t), \frac{1}{1.2} \leq t \leq 1, \\
\left({ }^{H} I_{\frac{1}{1.2}}^{0.7} y\right)\left(\frac{1}{1.2}^{+}\right) & =a \in \mathbb{R}^{2},
\end{aligned}
$$

where

$$
\mathbb{A}=\Theta, \quad \mathbb{B}=\left(\begin{array}{ll}
2 & 1 \\
3 & 5
\end{array}\right), \quad a=\left(\begin{array}{l}
1 \\
2
\end{array}\right) .
$$

The solution of (25) can be represented by $Y_{h, \alpha, \alpha}^{\Theta, \mathbb{B}}(t, s)$

$$
y(t)=Y_{h, \alpha, \alpha}^{\Theta, \mathbb{B}}(t, 1) a+\int_{1}^{h} Y_{h, \alpha, \alpha}^{\Theta, \mathbb{B}}(t, s) \mathbb{B} \varphi\left(\frac{s}{h}\right) \frac{d s}{s},
$$


where

$$
Y_{h, \alpha, \alpha}^{\Theta, \mathbb{B}}(t, s)= \begin{cases}0, & -\infty<t<s, \\ I, & t=s, \\ I \frac{(\ln t-\ln s)^{-0.7}}{\Gamma(0.3)}, & s<t \leq 1.2 s, \\ I \frac{(\ln t-\ln s)^{-0.7}}{\Gamma(0.3)}+\mathbb{B} \frac{((\ln t-\ln 1.2 s))^{-0.4}}{\Gamma(0.6)}, & 1.2 s<t \leq(1.2)^{2} s, \\ I \frac{(\ln t-\ln s)^{-0.7}}{\Gamma(0.3)}+\mathbb{B} \frac{((\ln t-\ln 1.2 s))^{-0.4}}{\Gamma(0.6)}+\mathbb{B}^{2} \frac{\left(\ln t-\ln (1.2)^{2} s\right)^{-0.1}}{\Gamma(0.9)} & (1.2)^{2} s<t \leq(1.2)^{3} s, \\ I \frac{(\ln t-\ln s)^{-0.7}}{\Gamma(0.3)}+\mathbb{B} \frac{((\ln t-\ln 1.2 s))^{-0.4}}{\Gamma(0.6)}+\mathbb{B}^{2} \frac{\left(\ln t-\ln (1.2)^{2} s\right)^{-0.1}}{\Gamma(0.9)} & \\ +\mathbb{B}^{3} \frac{\left(\ln t-\ln (1.2)^{3} s\right)^{0.2}}{\Gamma(1.2)} & (1.2)^{3} s<t \leq(1.2)^{4} s .\end{cases}
$$

Example 2. Consider

$$
\begin{aligned}
\left({ }^{H} D_{1+3}^{0.3} y\right)(t) & =\mathbb{B} y\left(\frac{t}{1.2}\right)+L_{f}\left(\begin{array}{c}
\sin y_{1}(t) \\
t \sin y_{2}(t)-y_{1}(t) \cos t
\end{array}\right), t \in(1,2.0736] \\
y(t) & =\varphi(t), \frac{1}{1.2} \leq t \leq 1 \\
\left({ }^{H}{ }_{\frac{1}{1.2}}^{0.7}+y\right)\left(\frac{1}{1.2}^{+}\right) & =a \in \mathbb{R}^{2} .
\end{aligned}
$$

Clearly, the function

$$
f(t, y)=L_{f}\left(\begin{array}{c}
\sin y_{1} \\
t \sin y_{2}-y_{1} \cos t
\end{array}\right)
$$

is jointly continuous and Lipschitz continuous with respect to $y$. We can choose $L_{f}>0$ so that the conditions of Theorems 3 and 4 are satisfied. Thus, the above problem has a unique solution which is Ulam-Hyers stable.

\section{Conclusions}

In this paper, we have introduced delayed perturbation of the M-L matrix exponential with logarithms, to get a representation formula for time-delay Hadamard type fractional differential equations with non-commutative linear part. Using this representation formula we have obtained several existence results for an initial value problem of time-delay Hadamard-type fractional differential equations. Furthermore, we have presented a sufficient condition for stability in the Ulam-Hyers sense. In our future work, we are planing to investigate the existence, stability and controllability of solutions to an initial value problem for time-delay fractional differential equations involving a combination of Caputo and Hadamard fractional derivatives.

Author Contributions: All authors contributed equally in this research paper. All authors have read and agreed to the published version of the manuscript.

Funding: This research received no external funding.

Conflicts of Interest: The authors declare no conflict of interest.

\section{References}

1. Diblík, J.; Fečkan, M.; Pospíšil, M. Representation of a solution of the Cauchy problem for an oscillating system with two delays and permutable matrices. Ukr. Math. J. 2015, 65, 58-69. [CrossRef]

2. Diblík, J.; Khusainov, D.Y.; Baštinec, J.; Sirenko, A.S. Exponential stability of linear discrete systems with constant coefficients and single delay. Appl. Math. Lett. 2016, 51, 68-73. [CrossRef] 
3. Khusainov, D.Y.; Shuklin, G.V. Linear autonomous time-delay system with permutation matrices solving. Stud. Univ. Žilina. 2003, 17, 101-108.

4. Medved', M.; Pospíšil, M. Representation of solutions of systems linear differential equations with multiple delays and linear parts given by nonpermutable matrices. J. Math. Sci. 2018, 228, 276-289. [CrossRef]

5. Pospíšil, M. Representation and stability of solutions of systems of functional differential equations with multiple delays. Electron. J. Qual. Theory Differ. Equ. 2012, 54, 1-30. [CrossRef]

6. Diblík, J.; Khusainov, D.Y. Representation of solutions of discrete delayed system $x(k+1)=A x(k)+B x(k-$ $-m)+f(k)$ with commutative matrices. J. Math. Anal. Appl. 2006, 318, 63-76. [CrossRef]

7. Diblík, J.; Khusainov, D.Y. Representation of solutions of linear discrete systems with constant coefficients and pure delay. Adv. Differ. Equ. 2006, 2006, 80825. [CrossRef]

8. Pospíšil, M. Representation of solutions of delayed difference equations with linear parts given by pairwise permutable matrices via Z-transform. Appl. Math. Comput. 2017, 294, 180-194.

9. Mahmudov, N.I. Representation of solutions of discrete linear delay systems with non permutable matrices. Appl. Math. Lett. 2018, 85, 8-14. [CrossRef]

10. Khusainov, D.Y.; Shuklin, G.V. Relative controllability in systems with pure delay. Int. J. Appl. Math. 2005, 2, 210-221. [CrossRef]

11. Medved', M.; Pospíšil, M.; Škripková, L. Stability and the nonexistence of blowing-up solutions of nonlinear delay systems with linear parts defined by permutable matrices. Nonlinear Anal. 2011, 74, 3903-3911. [CrossRef]

12. Medved', M.; Pospíšil, M. Sufficient conditions for the asymptotic stability of nonlinear multidelay differential equations with linear parts defined by pairwise permutable matrices. Nonlinear Anal. 2012, 75, 3348-3363. [CrossRef]

13. Li, M.; Wang, J.R. Finite time stability of fractional delay differential equations. Appl. Math. Lett. 2017, 64, 170-176. [CrossRef]

14. Li, M.; Wang, J.R. Exploring delayed M-L type matrix functions to study finite time stability of fractional delay differential equations. Appl. Math. Comput. 2018, 324, 254-265. [CrossRef]

15. Li, M.; Debbouche, A.; Wang, J.R. Relative controllability in fractional differential equations with pure delay. Math. Methods Appl. Sci. 2017, 41, 8906-8914. [CrossRef]

16. Liang, C.; Wang, J.R.; O'Regan, D. Representation of solution of a fractional linear system with pure delay. Appl. Math. Lett. 2018, 77, 72-78. [CrossRef]

17. Luo, Z.; Wang, J.R. Finite time stability analysis of systems based on delayed exponential matrix. J. Appl. Math. Comput. 2017, 55, 335-351. [CrossRef]

18. Mahmudov, N.I. Delayed perturbation of Mittag-Leffler functions and their applications to fractional linear delay differential equations. Math. Methods Appl. Sci. 2018, 42, 5489-5497. [CrossRef]

19. Mahmudov, N.I. A novel fractional delayed matrix cosine and sine. Appl. Math. Lett. 2019, 92, 41-48. [CrossRef]

20. Mahmudov, N.I. Fractional Langevin type delay equations with two fractional derivatives. Appl. Math. Lett. 2020, 103, 106215. [CrossRef]

21. Klimek, M. Sequential fractional differential equations with Hadamard derivative. Commun. Nonlinear Sci. Numer. Simul. 2011, 16, 4689-4697. [CrossRef]

22. Ma, Q.; Wang, R.; Wang, J.; Ma, Y. Qualitative analysis for solutions of a certain more generalized two-dimensional fractional differential system with Hadamard derivative. Appl. Math. Comput. 2014, 257, 436-445. [CrossRef]

23. Li M, Wang JR, Analysis of nonlinear Hadamard fractional differential equations via properties of Mittag-Leffler functions. J. Appl. Math. Comput. 2018, 51, 487-508.

24. Kilbas, A.A.; Srivastava, H.M.; Trujillo, J.J. Theory and Applications of Fractional Differential Equations. In North-Holland Mathematics Studies; Elsevier: Amsterdam, The Netherlands, 2006; Volume 204.

25. Ahmad, B.; Ntouyas S.K. Initial value problems of fractional order Hadamard-type functional differential equations. Electron. J. Differ. Equ. 2015, 77, 9.

26. Ahmad, B.; Ntouyas, S.K. A fully Hadamard type integral boundary value problem of a coupled system of fractional differential equations. Fract. Calc. Appl. Anal. 2014, 17, 348-360. [CrossRef]

27. Matar, M.A. Solution of sequential Hadamard fractional differential equations by variation of parameter technique. Abstr. Appl. Anal. 2018, 2018, 9605353. [CrossRef] 
28. Matar, M.; Al-Salmy, O.A. Existence and uniqueness of solution for Hadamard fractional sequential differential equations. IUG J. Nat. Stud. 2017, $3,141-147$.

29. Gambo, Y. Y.; Jarad, F.; Baleanu, D.; Abdeljawad, T. On Caputo modification of the Hadamard fractional derivatives. Adv. Differ. Equ. 2014, 2014, 10. [CrossRef]

30. Ahmad, B.; Ntouyas, S.K.; Agrawal, R.P.; Alsaedi, A. New results for boundary value problems of Hadamard-type fractional differential inclusions and integral boundary conditions. Bound. Value Probl. 2013, 2013, 275. [CrossRef]

31. Wang, G.; Pei, K.; Agrawal, R.P.; Zhang, L.; Ahmad, B. Nonlocal Hadamard fractional boundary value problem with Hadamard integral and discrete boundary conditions on a half-line. J. Comput. Appl. Math. 2018, 343, 230-239. [CrossRef]

32. Yukunthorn, W.; Ahmad, B.; Ntouyas, S.K.; Tariboon, J. On Caputo-Hadamard type fractional impulsive hybrid systems with nonlinear fractional integral conditions. Nonlinear Anal. Hybrid Syst. 2019, 19, 77-92. [CrossRef]

33. Yang, P.; Wang, J.R.; Zhou, Y. Representation of solution for a linear fractional delay differential equation of Hadamard type. Adv. Differ. Equ. 2019, 2019, 300. [CrossRef]

34. You, Z.; Fečkan, M.; Wang, J.R. Relative controllability of fractional delay differential equations via delayed perturbation of Mittag-Leffler functions. J. Comput. Appl. Math. 2020, 378, 112939. [CrossRef]

(C) 2020 by the authors. Licensee MDPI, Basel, Switzerland. This article is an open access article distributed under the terms and conditions of the Creative Commons Attribution (CC BY) license (http://creativecommons.org/licenses/by/4.0/). 